\title{
Application of Botulinum Toxin on Masticatory Muscle of Patients with Bruxism
}

\author{
Seok-Min Jang ${ }^{1}$, Hye-Mi Jeon ${ }^{1}$, Kyung-Hee Kim², Soo-Min Ok ${ }^{1}$, \\ Jun-Young Heo ${ }^{1}$, Sung-Hee Jeong ${ }^{1}$, Yong-Woo Ahn ${ }^{1}$ \\ 'Department of Oral Medicine, School of Dentistry, Pusan National University, Yangsan, Korea \\ ${ }^{2}$ Department of Oral Medicine, Busan Paik Hospital, Inje University College of Medicine, Busan, Korea
}

Received March 12, 2014 Revised March 28, 2014 Accepted April 17, 2014
Purpose: This study aims to evaluate the changes in soft tissue thickness of the masseteric region after injection of botulinum toxin type A (BTX-A).

Methods: Twenty-four data acquired from medical records were classified into 4 groups: bruxer group that received masseter muscle injection only (M-B), bruxer group that received both masseter and temporalis muscle injections (MT-B), non-bruxer group that received masseter muscle injection only (M-NB) and non-bruxer group that received both masseter and temporalis muscle injections (MT-NB). Injection dose of BTX-A was 30 units for each masseter muscle and 20 units for each temporalis muscle. We measured the reduced thickness of the masseteric region before and after 12 weeks after injection using cone-beam computed tomography.

Results: Among the patients that received both masseter and temporalis muscle injections, bruxer group showed a tendency to have more reduction in masseter muscle thickness than non-bruxer group. The difference in reduced thickness between M-B and MT-B tended to show greater than the difference between M-NB and MT-NB.

Conclusions: In case of masseter hypertrothy patients with bruxism there was a tendency to show a difference in reduced thickness of soft tissue between the group that received both masseter and temporalis muscles injection and the group that received masseter muscle injection only hence a thorough inspection before the injection of BTX-A is condisered to be needed.

Key Words: Botulinum toxins; Cone-beam computed tomography; Masticatory muscles

\section{INTRODUCTION}

Tooth clenching is continuously maintaining a static condition of maximum intercuspitation. This refers to the occlusal positioning of the mandible where complete intercuspation of the opposing teeth occurs. Teeth bruxing is a dynamic condition of maximum intercuspitation that refers to a forceful movement of the mandible from side to side.

The American Academy of Orofacial Pain has defined bruxism as the collective term for oral habits including clenching, bracing, gnashing and grinding of the teeth. It is a destructive and continuous habit which causes nonfunctional teeth wear and pain, periodontitis, masticatory muscle tension and pain, temporomandibular disorders (TMDs), neck and shoulder pain and headaches.

The treatment modalities for bruxism are psychotherapy (including stress management), biofeedback therapy and occlusal appliance therapy. Since Moore and Wood ${ }^{1)}$ and Smyth ${ }^{2)}$ reported bruxism reduction in their studies about masseteric hypertrophy, botulinum type A (BTX-A) has been used therapeutically for treatment of bruxism.

BTX-A is one of 8 toxins produced by clostridium botulinum. BTX-A attaches to the presynaptic nerve endings and plays a role in blocking muscle contraction by inhibiting the release of acetylcholine in the neuromuscular junction. The subunit with molecular weight of $50 \mathrm{kDa}$ in types B, D,

Copyright (c) 2014 Korean Academy of Orofacial Pain and Oral Medicine. All rights reserved. 
F, G acts at synaptobrevin; the subunit in types A, C, E acts at synaptosomal-associated protein; and the subunit in type $\mathrm{C}$ acts at syntaxin which results in the inhibition of the release of acetycholine. Among the 8 toxins, BTX-A shows the most potent effect and highest duration time. ${ }^{3)} \mathrm{New}$ nerve sprouts grow from 4 days after injection and form new neuromuscular synapses with the adjacent muscle hence temporarily restoring the role of parent terminals. ${ }^{4,5}$ After 4 weeks, parent terminals gradually recover and the function of the nerve sprouts reduce. After 3 months of the injection the original nerve endings are known to be fully recovered to the extent where they are indistinguishable from the ones before the injection.

There have been many previous studies about BTX-A application to patients with bruxism. Lee et al. ${ }^{6}$ reported that masseter muscle injection alone was effective whereas Guarda-Nardini et al. ${ }^{7)}$ showed both masseter and temporalis muscle injections were effective. There was also a study which had compared the effect of botulinum toxin injection with that of occlusal stabilization splint in bruxism patients. The study concluded that there was no difference in the effectiveness. In this study BTX-A was injected into masseter muscle only of the experimental group. ${ }^{8)}$ The other study compared the effect of BTX-A using electromyography (EMG) and both masseter and temporalis muscle of bruxism patients were injected in this study. ${ }^{9}$

Sohn et al. ${ }^{10)}$ investigated whether or not the presence of bruxism influences the treatment effect of the BTX-A injection into masseter muscle. They reported that regardless of the presence of bruxism, all subjects showed significantly reduced masseter muscle thickness at 3 months after injection and that bruxism may influence the recovery of atrophic masseter and decrease occlusal force due to BTX-A injection. There was a comparative analysis experiment with 4 groups that were classified by the injection sites of masseter only and both masseter and temporalis muscles and by the used materials of saline and BTX-A. It was found that a significant reduction in muscle fiber size was found in the group with BTX-A injection into both masseter and temporalis muscle. ${ }^{11}$

The previous studies about BTX-A application in bruxism evaluated its efficacy by injecting into masseter and temporalis muscles which are easy to approach externally among the masticatory muscles, but there was no study comparing the treatment effect of each. The aim of this study was to find out whether there was any difference in reduced thickness of soft tissue when BTX-A was injected into masseter only compared with when BTX-A was injected into both masseter and temporalis muscles and also the effect of each muscle injection on bruxism patients. This study is approved by the Institutional Review Board of Pusan National University Dental Hospital (PNUDH-2013-033).

\section{MATERIALS AND METHODS}

\section{Subjects}

We used the records of the patients who had visited at Goodwill Dental Hospital Deokcheon (Busan, Korea) from 2012 to 2013 with the chief complaint of masseteric hypertrophy and had received botulinum toxin treatment. The patients who received any other dental treatments were excluded except the patients wearing orthodontic appliances. The patients were recommended to receive both masseter and temporalis muscle injections but the injection was given into masseter only or into both masseter and temporalis muscles according to their choices. The subjects were all women and none of them had known systemic disease, pregnancy, possible pregnancy or any other specific medical history and were not taking any regular medications such as anti-depressants. Age ranged from 22 to $41(25.21 \pm 4.42)$ years.

\section{Methods}

The preliminary questionnaires were done and the followings were included in bruxism section: (1) no bruxism, (2) patients were unaware about their bruxism but happened to be told by others, and (3) self-awareness of bruxism. The patients who answered no bruxism were classified into NB group while the patients who answered that they had bruxism into B group. The patients were also classified according to the botulinum toxin injection sites and $\mathrm{M}$ group received masseter injection only whereas MT group received both masseter and temporalis injections. The thickness of soft tissue at first visit and after botulinum toxin injection were compared.

BTX-A, Meditoxin (Medytox, Cheongwon, Korea) which 
contained 100 units of vacuum-freeze dried BTX-A per one vial was diluted with $1.0 \mathrm{~mL}$ of sterile, non-preserved normal saline (0.9\% sodium chloride) to a concentration of 10.0 unit of BTX-A per $0.1 \mathrm{~mL}$.

Insulin syringe of $0.5 \mathrm{~mL}$ was used. The 3 injection sites corresponded to area of greatest muscle mass in lower third of each masseter muscle were marked and injected and the distance between each site was about $1 \mathrm{~cm}$. In case of temporalis muscle, 5 sites from anterior and middle parts of temporalis muscle were marked and injected each side. Thirty units of BTX-A was injected into each masseter muscle of M group. For MT group patients, 30 units of BTX-A was injected into each masseter muscle as well as 20 units of BTX-A into each temporalis muscle. Cone-beam computed tomography (CBCT) was taken in a relaxed condition of masticatory muscle before and 12 weeks after injection.

Acquired images of CBCT before and after injection were reconstructed and analyzed using Invivo5 (Anatomage, San

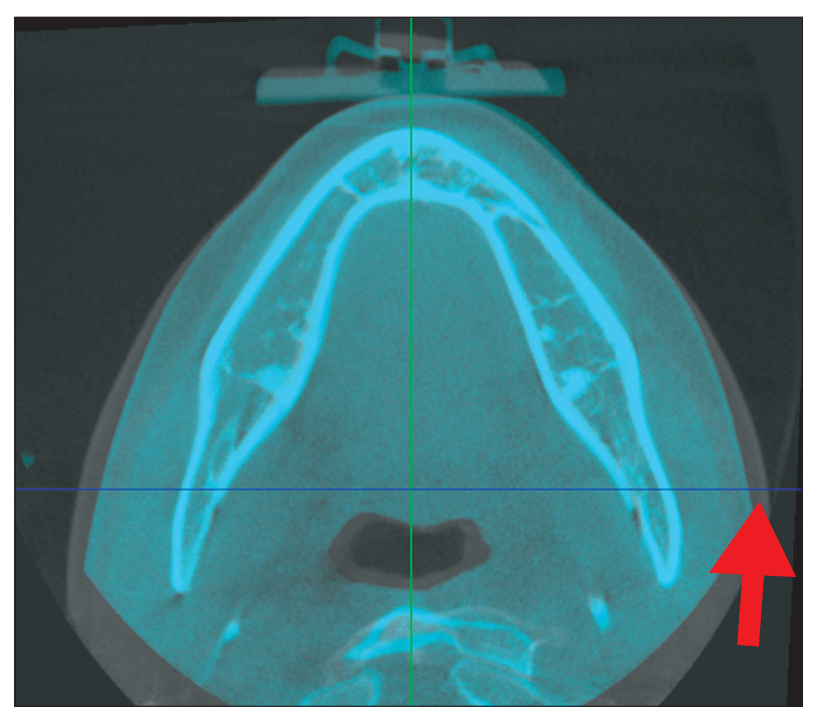

Fig. 1. Superimposed cone-beam computed tomography image and measurement of soft tissue changes (arrow).
Jose, CA, USA) and the point which were thought to be the nearest to the injection site was marked at the position where the line drawn from the center of coronoid process crossed the center of mandibular body and measured.

Images before and after injection were superimposed and the position to be measured was superimposed as precisely as possible to reduce the error made by the position of measurement to a minimum (Fig. 1). Total lengths of the images were measured on the basis of the axis. The distances between the external surfaces of the mandible were also measured and the differences between the two sets of measurements were used as the results.

Statistical analysis of the collected data was done using IBM SPSS Statistics 20.0 (IBM Co., Armonk, NY, USA). Normality test was done and independent t-test was performed. Significant level was 5\% in this study $(\mathrm{p}<0.05)$.

\section{RESULTS}

\section{Comparison between $M$ group and MT group}

The classification for this study were as follows (Table 1). The measurements for M group (Table 2) and MT group (Table 3) were as follows.

The average reduced thickness of soft tissue in the subjects was $4.11 \mathrm{~mm}$ and in comparison of the reduced thickness of masseter muscle according to injection sites, the group that received both masseter and temporalis muscle injections tended to show slightly more reduction in masseter muscle thickness than the group that received masseter muscle injection only (Table 4).

\section{Comparison according bruxism}

In comparison of soft tissue width between bruxer group

Table 1. Classification for study

\begin{tabular}{|c|c|c|}
\hline \multicolumn{2}{|c|}{ Classification (group) } & \multirow{2}{*}{$\begin{array}{c}\begin{array}{c}\text { Subject } \\
\text { (No.) }\end{array} \\
6\end{array}$} \\
\hline $\mathrm{M}-\mathrm{NB}$ & Non-bruxism patients injected into masseter muscle & \\
\hline MT-NB & Non-bruxism patients injected into masseter and temporalis muscle & 7 \\
\hline$M-B$ & Bruxism patients injected into masseter muscle & 6 \\
\hline MT-B & Bruxism patients injected into masseter and temporalis muscle & 5 \\
\hline \multicolumn{2}{|l|}{ Total } & 24 \\
\hline \multicolumn{2}{|c|}{ Age $(y)$, mean \pm standard deviation } & $25.21 \pm 4.42$ \\
\hline
\end{tabular}


Table 2. The changes in soft tissue thickness after botulinum toxin injection in M group ( $\mathrm{mm}$ )

\begin{tabular}{cccc}
\hline M & S1 & S2 & S1-S2 \\
\hline 1 & 37.29 & 33.76 & 3.53 \\
2 & 39.15 & 35.17 & 3.98 \\
3 & 39.59 & 34.36 & 5.23 \\
4 & 29.63 & 27.93 & 1.70 \\
5 & 37.33 & 33.87 & 3.46 \\
6 & 39.73 & 35.04 & 4.69 \\
7 & 35.00 & 30.55 & 4.45 \\
8 & 41.68 & 39.50 & 2.18 \\
9 & 37.10 & 31.71 & 5.39 \\
10 & 33.19 & 29.53 & 3.66 \\
11 & 41.01 & 34.43 & 6.58 \\
12 & 32.70 & 28.89 & 3.81 \\
Mean \pm SD & $36.95 \pm 3.67$ & $32.90 \pm 3.28$ & $4.06 \pm 1.35$ \\
\hline
\end{tabular}

$M$, the group received masseter muscle injection only; S1, soft tissue width at first visit; S2, soft tissue width after 12 weeks; S1-S2, reduced thickness from first visit until 12 weeks after; SD, standard deviation.

Table 3. The changes in soft tissue thickness after botulinum toxin injection in MT group (mm)

\begin{tabular}{cccc}
\hline MT & S1 & S2 & S1-S2 \\
\hline 1 & 30.66 & 27.35 & 3.31 \\
2 & 28.03 & 24.16 & 3.87 \\
3 & 36.52 & 29.17 & 7.35 \\
4 & 40.24 & 35.43 & 4.81 \\
5 & 33.36 & 31.15 & 2.21 \\
6 & 44.69 & 41.18 & 3.51 \\
7 & 32.43 & 28.82 & 3.61 \\
8 & 35.28 & 31.59 & 3.69 \\
9 & 25.64 & 23.26 & 2.38 \\
10 & 36.87 & 30.68 & 6.19 \\
11 & 37.91 & 32.53 & 5.38 \\
12 & 37.02 & 33.44 & 3.58 \\
Mean \pm SD & $34.89 \pm 5.26$ & $30.73 \pm 4.85$ & $4.16 \pm 1.52$ \\
\hline
\end{tabular}

MT, the group received both masseter and temporalis muscle injections; S1, soft tissue width at first visit; S2, soft tissue width after 12 weeks; S1-S2, reduced thickness from first visit until 12 weeks after; $\mathrm{SD}$, standard deviation.

and non-bruxer group at first visit, the bruxer group showed a tendency to have greater soft tissue width (Table 5).

Among the patients in the group that received masseter muscle injection only, the non-bruxer group tended to show more reduction in masseter muscle thickness (Table 6).

Among the patients in the group that received both masseter and temporalis muscle injections, the bruxer group tended to report more reduction in masseter muscle thickness (Table 7).

In comparison between respective bruxer groups within
Table 4. Comparison of the reduced thickness of masseter muscle in $\mathrm{M}$ group and MT group at 12 weeks after botulinum toxin injection $(\mathrm{mm})$

\begin{tabular}{cccc}
\hline & $\mathrm{M}$ & $\mathrm{MT}$ & $\mathrm{p}$-value \\
\hline S1-S2 & $4.06 \pm 1.35$ & $4.16 \pm 1.52$ & 0.863 \\
\hline
\end{tabular}

$M$, the group received masseter muscle injection only; $M T$, the group received both masseter and temporalis muscle injections.

Statistics by independent two samples t-test.

Values are presented as mean \pm standard deviation.

Table 5. Soft tissue width in B group and NB group at first visit (mm)

\begin{tabular}{cccc}
\hline & B & NB & $p$-value \\
\hline S1 & $37.39 \pm 3.63$ & $34.68 \pm 5.02$ & 0.151 \\
\hline
\end{tabular}

$B$, group of bruxism patients; NB, group of non-bruxism patients; S1, soft tissue width at first visit.

Statistics by independent two samples t-test.

Values are presented as mean \pm standard deviation.

Table 6. The reduced thickness of masseter muscle in M-NB group and M-B group at 12 weeks after botulinum toxin injection ( $\mathrm{mm}$ )

\begin{tabular}{cccc}
\hline & M-NB & M-B & p-value \\
\hline S1-S2 & $4.18 \pm 0.88$ & $3.93 \pm 1.78$ & 0.765 \\
\hline
\end{tabular}

S1-S2, reduced thickness from first visit until 12 weeks after; M-NB, non-bruxer group who received masseter muscle injection only; $M-B$, bruxer group who received masseter muscle injection only. Statistics by independent two samples t-test.

Values are presented as mean \pm standard deviation.

Table 7. The reduced thickness of masseter muscle in MT-NB group and MT-B group at 12 weeks after botulinum toxin injection ( $\mathrm{mm}$ )

\begin{tabular}{cccc}
\hline & MT-NB & MT-B & p-value \\
\hline S1-S2 & $3.79 \pm 1.16$ & $4.67 \pm 1.93$ & 0.350 \\
\hline
\end{tabular}

S1-S2, reduced thickness from first visit until 12 weeks after; MT$\mathrm{NB}$, non-bruxer group who received both masseter and temporalis muscle injections; MT-B, bruxer group who received both masseter and temporalis muscle injections.

Statistics by independent two samples t-test.

Values are presented as mean \pm standard deviation.

Table 8. The reduced thickness of masseter muscle in M-B group and MT-B group at 12 weeks after botulinum toxin injection $(\mathrm{mm})$

\begin{tabular}{cccc}
\hline & M-B & MT-B & p-value \\
\hline S1-S2 & $3.93 \pm 1.78$ & $4.67 \pm 1.93$ & 0.528 \\
\hline
\end{tabular}

S1-S2, reduced thickness from first visit until 12 weeks after; $M-B$, bruxer group who received masseter muscle injection only; MT-B, bruxer group who received both masseter and temporalis muscle injections.

Statistics by independent two samples t-test.

Values are presented as mean \pm standard deviation. 
the groups that received masseter muscle injection only and the group that received both masseter and temporalis muscle injections, the group that received both masseter and temporalis muscle injections showed a tendency to have more reduction in masseter muscle thickness than the group that received masseter muscle injection only (Table 8).

\section{DISCUSSION}

Bruxism can be defined as the action of non-functional clenching or grinding of the teeth during day or night. Bruxism is usually limited to the cases of nocturnal, unconscious habit but it can appear during daytime both consciously and unconsciously. Non-functional activities including pencil, nail, cheek or lip biting can appear consciously. Chewing gum continuously also can be included in non-functional activity. The etiology of bruxism is uncertain but it is known to be related to occlusal disturbance, genetic factors, allergy, central nervous system disorders and psychological factors in general. ${ }^{12-14)}$

The prevalence of generically identified "bruxism" are reported to range from $8 \%$ to $31.4 \%$. The prevalence of awake bruxism is $22.1 \%$ to $31 \%$ and the prevalence of sleep bruxism is found to be more consistent with $12.8 \% \pm 3.2 \%$. Bruxism activities are found to be unrelated to sex, and a decrease with age is described in elderly people. ${ }^{15)}$ In modern times, emotional stress is considered to be the main factor influencing bruxism activity. Stress from psychosocial factors leads to muscle tension and awakens bruxism in order to reduce this increased muscle activities. ${ }^{16)}$ In the early studies, the etiology of bruxism was thought to be related directly to occlusal discrepancies, however, recent studies are reporting that bruxism originates from the central nervous system. ${ }^{17)}$

The severity of bruxism also tends to increase with the level of stress perceived by the individual. The level of perceived stress from the daytime is found to be associated with the severity of nocturnal bruxism on the day. Persistent bruxism can cause hyperactivity or discrepancy in masticatory muscles and internal derangement of temporomandibular joint and it can have an influence on myofacial pain or tension type headache. ${ }^{18,19)}$

Psychological therapy (including stress management), biofeedback therapy and occlusal appliance therapy can be included in the management of bruxism. ${ }^{20)}$

BTX-A which is used in the treatment of masseteric hypertrophy with bruxism recently was used for the first time to treat strabismus by Scott ${ }^{21)}$ and the Food and Drug Administration officially approved its use for the treatment of blepharospam, strabismus and diseases related to facial nerve in 1989. Since then Moore and Wood ${ }^{1)}$ and Smyth ${ }^{2)}$ reported that BTX-A showed an effect on the reduction of bruxism in their studies about masseteric hypertrophy, it has been used therapeutically in dentistry. In 1999, Freund et al. ${ }^{4)}$ reported the efficacy of BTX-A treatment for TMD.

Nowadays, BTX-A is used to treat chronic masticatory muscle pain such as myofacial pain, bruxism, masseter hypertrophy and mandibular dyskinesia etc. in facial region. ${ }^{22)}$ BTX-A can be applied in the treatment of headaches and its prevention as well as known to be effective in migraine and tension-type headaches. ${ }^{23)}$ Sidebottom et al. ${ }^{24)}$ measured pain scores using visual analogue scale before and after BTX-A injection in TMD patients and reported that there was 52.8\% improvement in pain score in masseter muscle injected patients whereas 56.9\% improvement in temporalis muscle injected patients.

Nowadays BTX-A has various indications such as strabismus, blepharospam, cervical dystonia, upper limb spasticity, chronic migraine, ${ }^{23,25,26)}$ hyperhidrosis and urinary incontinence etc. ${ }^{22)}$ BTX-A is contraindicated in patients afflicted with a preexisting motor neuron disorders like myasthenia gravis, patients who are hypersensitive to any of the components in BTX-A, patients taking or possible to take aminoglycisides (tobramycin, gentamicin) or spectinomycin, pregnancy and lactating females. Relative contraindications include patients with acute infectious disease, liver disease, heart disease and lung disease, active tuberculosis patients and patients with blood disease.

There were some studies about BTX-A application in management of bruxism. BTX-A was injected into masseter and temporalis muscles of the patient who showed severe bruxism recovering from a coma ${ }^{27)}$ and the patient who showed bruxism after anoxic brain injury secondary to cardiac arrest and observed dramatic reduction in bruxism. ${ }^{28)}$ There was also a report about the use of BTX-A to treat masticatory muscle spasm due to an increase in intracranial 
pressure after accident. ${ }^{29)}$

According to Amorim et al. ${ }^{30)}$ bruxism patients showed increased muscle activities in both masseter and temporalis muscles. The temporalis is a postural muscle controlling mandibular movements in excursive function and temporalis muscle activity tends to increase when masseter muscle activity decreases.

von Lindern et al. ${ }^{31)}$ reported the reduction in the masseter muscles after BTX-A injection into both masseters of masseter hypertrophy patients. Van Zandijcke and Marchau ${ }^{32}$ reported that BTX-A was injected into masseter and temporalis muscles of the patient who showed severe bruxism recovering from a coma and observed a marked reduction in the bruxism. Ivanhoe et al. ${ }^{28)}$ reported that severe bruxism of brain injury patient could be temporarily stopped with BTX-A. Reduction in bruxism, change in headache symptoms and reduction in masseter area could also be observed in this study according to the subjective reports of the patients.

To et al. ${ }^{33)}$ measured the volume reduction in masseter muscle using ultrasonography and EMG after BTX-A injection into both masseter muscle and reported about 31\% volume reduction in masseter muscle at 3 months after injection. Kim et al. ${ }^{34)}$ reported about $22 \%$ volume reduction measured from CT images at 3 months after injection. However, our study showed only $11.4 \% \pm 0.71 \%$ reduction. The lower percentage results may be due to the fact that soft tissue like masseter muscle, fat and skin were not distinguishable in $\mathrm{CBCT}$ images, hence the measurements in our study made from the external surface of mandible to the epidermis was the total thickness of soft tissue, not the exact thickness of masseter muscle in a relaxed condition.

For the treatment of masseter hypertrophy, BTX-A injection into temporalis muscles are not included in general. According to our study's results there was no significant difference found in reduced thickness of facial soft tissue between the group that received masseter muscle injection only and the group that received both masseter and temporalis injections. Therefore injection into masseter muscle only is thought to be reasonably sufficient when BTX-A is injected for esthetic reasons. However, in the case of masseter hypertrophy patients with bruxism, the group that received both masseter and temporalis muscle injections tended to show more reduction in soft tissue thickness even though the results were not significant. Therefore, both masseter and temporalis muscle injections are needed to be considered in treatment of masseter hypertrophy patients with bruxism.

The thickness of soft tissue in bruxer group tended to be thicker than that of non-bruxer group but there was no significant difference in statistics.

In comparison among 4 groups classified by their injection sites and the presence of bruxism, the bruxer group tended to show a big difference in reduced thickness of soft tissue according to injection sites compared to the nonbruxer group. Further studies in larger sample size are considered to be necessary.

This study has not considered TMD including headache, osteoarthritis, muscle pain and disc displacement etc. Therefore further studies about TMD patients group are thought to be needed.

This study compared the difference in reduced thickness of soft tissue when BTX-A was injected into masseter only and when BTX-A was injected into both masseter and temporalis muscles and also their effect on bruxism and obtained the following results.

In comparison of the reduced thickness of masseter muscle according to injection sites, the group that received both masseter and temporalis muscle injections tended to show slightly more reduction in masseter muscle thickness than the group that received masseter muscle injection only but we could notice that there was not much difference in their effect. In comparison of soft tissue width between bruxer group and non-bruxer group at first visit, bruxer group showed a tendency to have greater soft tissue width. This implies that bruxism can have an effect on the increase in soft tissue thickness. Among the patients that received masseter muscle injection only, non-bruxer group showed a tendency to have slightly more reduction in masseter muscle thickness than bruxer group but the difference was small.

Among the patients that received both masseter and temporalis muscle injections, bruxer group showed a tendency to have more reduction in masseter muscle thickness than non-bruxer group. The difference in reduced thickness between the group that received masseter muscle injection 
only and the group that received both masseter and temporalis muscle injections among bruxers tended to show greater than the difference between the two groups among non-bruxers. This implies that the correction of both masseter and temporalis muscle have an effect on bruxism.

Our study confirmed that there was only a small difference found in the reduced thickness in facial soft tissue between the group that received masseter muscle injection only and the group that received both masseter and temporalis injections and concluded that the injection into masseter muscle only was thought to be reasonably sufficient to get a satisfying result when BTX-A was injected for esthetic reasons. However in the case of masseter hypertrophy patients with bruxism, there was a tendency to show a difference in reduced thickness of soft tissue between the group that received both masseter and temporalis muscles injection and the group that received masseter muscle injection only hence a thorough inspection before the injection of BTX-A is considered to be needed and further studies are considered to be required.

\section{CONFLICT OF INTEREST}

No potential conflict of interest relevant to this article was reported.

\section{REFERENCES}

1. Moore AP, Wood GD. The medical management of masseteric hypertrophy with botulinum toxin type A. Br J Oral Maxillofac Surg 1994;32:26-28.

2. Smyth AG. Botulinum toxin treatment of bilateral masseteric hypertrophy. Br J Oral Maxillofac Surg 1994;32:29-33.

3. Nigam PK, Nigam A. Botulinum toxin. Indian J Dermatol 2010; 55:8-14.

4. Freund B, Schwartz M, Symington JM. The use of botulinum toxin for the treatment of temporomandibular disorders: preliminary findings. J Oral Maxillofac Surg 1999;57:916-920; discussion 920-921.

5. Niamtu J 3rd. Aesthetic uses of botulinum toxin A. J Oral Maxillofac Surg 1999;57:1228-1233.

6. Lee SJ, McCall WD Jr, Kim YK, Chung SC, Chung JW. Effect of botulinum toxin injection on nocturnal bruxism: a randomized controlled trial. Am J Phys Med Rehabil 2010;89:16-23.

7. Guarda-Nardini L, Manfredini D, Salamone M, Salmaso L, Tonello S, Ferronato G. Efficacy of botulinum toxin in treating myofascial pain in bruxers: a controlled placebo pilot study. Cranio 2008;26:126-135.
8. Long H, Liao Z, Wang Y, Liao L, Lai W. Efficacy of botulinum toxins on bruxism: an evidence-based review. Int Dent J 2012;62: $1-5$.

9. Clark GT, Ram S. Four oral motor disorders: bruxism, dystonia, dyskinesia and drug-induced dystonic extrapyramidal reactions. Dent Clin North Am 2007;51:225-243.

10. Sohn SM, Chung GC, Kim ME, Kim KS. Nocturnal bruxism and botulinum toxin effect on the subjects with masseteric hypertrophy. Korean J Oral Med 2007;32:337-346.

11. Tsai CY, Lin YC, Su B, Yang LY, Chiu WC. Masseter muscle fibre changes following reduction of masticatory function. Int J Oral Maxillofac Surg 2012;41:394-399.

12. [No authors listed]. The glossary of prosthodontic terms. J Prosthet Dent 2005;94:10-92.

13. American Academy of Sleep Medicine. The international classification of sleep disorders: diagnostic \& coding manual. 2nd ed. Westchester: American Academy of Sleep Medicine; 2005.

14. De Leeuw R. Orofacial pain: guidelines for assessment, diagnosis, and management. 4th ed. Chicago: Quintessence Publishing; 2008. pp. 316.

15. Manfredini D, Winocur E, Guarda-Nardini L, Paesani D, Lobbezoo F. Epidemiology of bruxism in adults: a systematic review of the literature. J Orofac Pain 2013;27:99-110.

16. Ahlberg J, Lobbezoo F, Ahlberg K, et al. Self-reported bruxism mirrors anxiety and stress in adults. Med Oral Patol Oral Cir Bucal 2013;18:e7-e11.

17. Lobbezoo F, Naeije M. Bruxism is mainly regulated centrally, not peripherally. J Oral Rehabil 2001;28:1085-1091.

18. Kalaykova SI, Lobbezoo F, Naeije M. Risk factors for anterior disc displacement with reduction and intermittent locking in adolescents. J Orofac Pain 2011;25:153-160.

19. Alves AC, Alchieri JC, Barbosa GA. Bruxism. Masticatory implications and anxiety. Acta Odontol Latinoam 2013;26:15-22.

20. Lobbezoo F, van der Zaag J, van Selms MK, Hamburger HL, Naeije M. Principles for the management of bruxism. J Oral Rehabil 2008;35:509-523.

21. Scott $A B$. Botulinum toxin injection of eye muscles to correct strabismus. Trans Am Ophthalmol Soc 1981;79:734-770.

22. Rao LB, Sangur R, Pradeep S. Application of Botulinum toxin type A: an arsenal in dentistry. Indian J Dent Res 2011;22:440445.

23. Silberstein S, Mathew N, Saper J, Jenkins S. Botulinum toxin type A as a migraine preventive treatment. For the BOTOX Migraine Clinical Research Group. Headache 2000;40:445-450.

24. Sidebottom AJ, Patel AA, Amin J. Botulinum injection for the management of myofascial pain in the masticatory muscles. A prospective outcome study. Br J Oral Maxillofac Surg 2013;51: 199-205.

25. Binder WJ, Brin MF, Blitzer A, Schoenrock LD, Pogoda JM. Botulinum toxin type A (BOTOX) for treatment of migraine headaches: an open-label study. Otolaryngol Head Neck Surg 2000; 123:669-676.

26. Schulte-Mattler WJ, Wieser T, Zierz S. Treatment of tension-type headache with botulinum toxin: a pilot study. Eur J Med Res 1999;4:183-186.

27. El Maaytah M, Jerjes W, Upile T, Swinson B, Hopper C, Ayliffe P. 
Bruxism secondary to brain injury treated with botulinum toxinA: a case report. Head Face Med 2006;2:41.

28. Ivanhoe CB, Lai JM, Francisco GE. Bruxism after brain injury: successful treatment with botulinum toxin-A. Arch Phys Med Rehabil 1997;78:1272-1273.

29. Pidcock FS, Wise JM, Christensen JR. Treatment of severe posttraumatic bruxism with botulinum toxin-A: case report. J Oral Maxillofac Surg 2002;60:115-117.

30. Amorim CF, Vasconcelos Paes FJ, de Faria Junior NS, de Oliveira LV, Politti F. Electromyographic analysis of masseter and anterior temporalis muscle in sleep bruxers after occlusal splint wearing. J Bodyw Mov Ther 2012;16:199-203.

31. von Lindern JJ, Niederhagen B, Bergé S, Appel T. Type A botuli- num toxin in the treatment of chronic facial pain associated with masticatory hyperactivity. J Oral Maxillofac Surg 2003;61:774778.

32. Van Zandijcke M, Marchau MM. Treatment of bruxism with botulinum toxin injections. J Neurol Neurosurg Psychiatry 1990;53:530.

33. To EW, Ahuja AT, Ho WS, et al. A prospective study of the effect of botulinum toxin A on masseteric muscle hypertrophy with ultrasonographic and electromyographic measurement. Br J Plast Surg 2001;54:197-200.

34. Kim HJ, Yum KW, Lee SS, Heo MS, Seo K. Effects of botulinum toxin type A on bilateral masseteric hypertrophy evaluated with computed tomographic measurement. Dermatol Surg 2003;29: 484-489. 